\title{
Geometry of the Computational Singular Perturbation Method
}

\author{
Hans G. Kaper ${ }^{1}$, Tasso J. Kaper ${ }^{*}$, Antonios Zagaris ${ }^{3}$ \\ ${ }^{1}$ Department of Mathematics and Statistics, Georgetown University \\ Washington, D.C. 20057, USA \\ 2 Department of Mathematics and Statistics, Boston University \\ Boston, MA 02215, USA \\ 3 Applied Analysis and Mathematical Physics \\ University of Twente, 7522NB Enschede, the Netherlands
}

\begin{abstract}
The Computational Singular Perturbation (CSP) method, developed by Lam and Goussis [Twenty-Second Symposium (International) on Combustion, The Combustion Institute, Pittsburgh, 1988, pp. 931-941], is a commonly-used method for finding approximations of slow manifolds in systems of ordinary differential equations (ODEs) with multiple time scales. The validity of the CSP method was established for fast-slow systems with a small parameter $\varepsilon$ by the authors in [Journal of Nonlinear Science, 14 (2004), 59-91]. In this article, we consider a more general class of ODEs which lack an explicit small parameter $\varepsilon$, but where fast and slow variables are nevertheless separated by a spectral gap. First, we show that certain key quantities used in the CSP method are tensorial and thus invariant under coordinate changes in the state space. Second, we characterize the slow manifold in terms of these key quantities and explain how these characterizations are related to the invariance equation. The implementation of the CSP method can be either as a one-step or as a two-step procedure. The one-step CSP method aims to approximate the slow manifold; the two-step CSP method goes one step further and aims to decouple the fast and slow variables at each point in the state space. We show that, in either case, the operations of changing coordinates and performing one iteration of the CSP method commute. We use the commutativity property to give a new, concise proof of the validity of the CSP method for fast-slow systems and illustrate with an example due to Davis and Skodje.
\end{abstract}

Dedicated to Professor Alexander Gorban on the occasion of his sixtieth birthday

Keywords and phrases: nonlinear differential equations, multiple time scales, model reduction, chemical kinetics, slow manifold, dimension reduction, computational singular perturbation method, spectral gap

Mathematics Subject Classification: 34C20, 80A30, 92C45, 34E13, 34E15, 80A25

${ }^{*}$ Corresponding author. E-mail: tasso@math.bu.edu 


\section{Introduction}

This article is concerned with the Computational Singular Perturbation (CSP) method, a dimension reduction method for systems of autonomous ordinary differential equations (ODEs) with multiple time scales. The systems we consider are assumed to have an invariant, low-dimensional manifold. Solutions evolve on a fast time scale toward a neighborhood of that manifold; once there, they stay in the neighborhood and evolve on a much slower time scale following the dynamics on it. Because the motion in that neighborhood is slow relative to the motion away from it, the manifold is commonly referred to as a "slow manifold" and the dynamics on it as "slow dynamics."

Since the dimension of the slow manifold is less than that of the state space, one can achieve significant savings in the study of the long-term behavior of such systems by considering the slow dynamics. Many techniques have been proposed for this purpose, especially in the chemical kinetics literature; see for example the references in $[6-10,12,36,38]$. Generally, these reduction methods involve the construction of a coordinate system where the slow manifold has a simple functional representation - the ideal system being one where a number of components (the "fast" components) of the state vector are identically zero, as in the Fenichel normal form [5,18]. The CSP method differs from these reduction methods in that it focuses on the dynamics of the vector field itself, rather than on the dynamics of the state variables.

The CSP method was first proposed by Lam and Goussis [24] and subsequently developed in [11,22,23, $25,26]$. It is being used widely, for example, in combustion modeling [1,2,14-16,28,29,32-35], atmospheric science $[27,30]$ and biology $[13,20,21]$. It is an iterative technique to generate a local coordinate system in which to express the vector field $g$. Written in the vector basis $\left\{A_{1}(x), \ldots, A_{N}(x)\right\}$ for $T_{x} \mathbb{R}^{N}$, the vector field acquires new amplitudes $f=\left\{f_{1}, \ldots, f_{N}\right\}$ by means of the identity $g(x)=\sum_{i=1}^{N} A_{i}(x) f_{i}(x)$. The CSP method is underpinned by the nonlinear dynamics in the tangent bundle of these amplitudes, which are generated by a Lie bracket $\Lambda$ involving the vector field itself. At each iteration, a transformation of the vector basis is introduced to reflect more accurately the fast-slow decomposition of the equations at each point in state space. This transformation of bases may be implemented either as a one-step procedure or as a two-step procedure. In the one-step procedure, the aim is to approximate the slow manifold by successively transforming the basis of the slow subspace at each point so that it becomes independent of the fast variables. In the two-step procedure, one successively transforms the bases of both the slow and fast subspaces, so that one not only approximates the slow manifold but also decouples the fast and slow variables at each point in the state space.

The method has been analyzed mathematically [36,37], and its approximation properties have been established rigorously for fast-slow systems of the form

$$
\begin{aligned}
& x_{1}^{\prime}=g_{1}\left(x_{1}, x_{2}\right), \\
& x_{2}^{\prime}=\varepsilon g_{2}\left(x_{1}, x_{2}\right) .
\end{aligned}
$$

In these systems, $\left(x_{1}, x_{2}\right): t \mapsto\left(x_{1}(t), x_{2}(t)\right) \in \mathbb{R}^{N_{1}} \oplus \mathbb{R}^{N_{2}}$ are a pair of functions and $\varepsilon$ an arbitrarily small positive parameter.

In this article, we consider the CSP method for a more general class of ODEs for a vector-valued function $x: t \mapsto x(t) \in \mathbb{R}^{N}$,

$$
x^{\prime}=g(x),
$$

where there is no explicit small parameter $\varepsilon$, but where the spectrum of the Jacobian $D_{x} g$ consists of two disjoint parts separated by a spectral gap,

$$
\Re\left(\lambda_{1}\right) \leq \cdots \leq \Re\left(\lambda_{N_{1}}\right) \leq \lambda_{f}<\lambda_{s} \leq \Re\left(\lambda_{N_{1}+1}\right) \leq \cdots \leq \Re\left(\lambda_{N}\right)<0,
$$

for some real numbers $\lambda_{f}$ and $\lambda_{s}$.

This type of spectral gap condition is natural for systems (1.2) that possess invariant manifolds. It holds in large classes of systems to which model reduction is applied. Generally, in these systems, the components of $x$ all evolve on both the slow and the fast time scales, with the evolution being dominated by 
the fast scales away from a lower-dimensional invariant manifold and by the slow scales near it. Without loss of generality for the analysis of the CSP method, we consider systems in which the slow manifold is parametrized by the vector $x_{2}$ and has dimension $N_{2}=N-N_{1}$, and we note that implementation of the CSP method does not a priori require this this parametrization, see [11,22,24-26]. Moreover, we observe that fast-slow systems are a special case, where the small parameter $\varepsilon$ is inversely proportional to the width of the spectral gap and certain state variables evolve slowly, see for example [14,36,37].

Our analysis here relies, first, on the existence of normal forms common to systems (1.2) and, second, on the tensorial (coordinate-free) nature of the CSP method. In particular, each CSP iteration produces identical results irrespectively of the state space coordinates in which it is carried out, and hence its effectiveness may be assessed in a coordinate system of choice.

This article is organized as follows. In Sections 2 and 3, we formulate the problem (1.2) and review the spectral gap condition and the dynamics of the vector field $g$ in the tangent bundle. In Section 4 , we show that the amplitudes $f$ and operator $\Lambda$ are invariant under coordinate changes in the state space. In Section 5 , we briefly review the fast-slow decomposition of $\Lambda$ that is induced by the spectral gap condition. Then, in Section 6, we show that the slow manifold is characterized by a simple condition on one of the components of $\Lambda$, namely a component that governs the coupling of the fast amplitudes to the slow ones. In Section 7, we focus on the one-step procedure and show that the operations of changing state space coordinates and performing a CSP iteration commute. Also, we show how successive iterates improve the accuracy of the approximation of the slow manifold. Next, in Section 8, we use the commutativity property to give a new, concise proof of the validity of the CSP method for fast-slow systems with an explicit small parameter. This concise proof is modular, considerably shorter than the original proof of validity using the method of induction in [36], and it sheds new light on the method. In Section 9, an example due to Davis and Skodje is used to illustrate the application of the one-step procedure. Finally, in Section 10, we extend all of the main results - established in Sections 7-8 for the one-step CSP method - to the full, two-step CSP method. For notational simplicity, we have uniformly dispensed with the distinction between sub- and superscripts for the coordinates $x$, as well as for tensorial quantities. As a result, all indices are denoted by subscripts, irrespectively of whether they characterize the components of vectors or co-vectors.

\section{Fast-Slow Dynamics}

We let $g: \mathbb{R}^{N} \rightarrow \mathbb{R}^{N}$ be a given, smooth vector field and consider the autonomous ODE

$$
x^{\prime}=g(x)
$$

for the vector-valued function $x: t \mapsto x(t) \in \mathbb{R}^{N}$. Here, a prime ' denotes differentiation with respect to $t$. In principle, this equation contains the entire system dynamics: the evolution of other dynamic quantities is contingent on and follows from it. The evolution of the components of $g$, in particular, is described by the ODE

$$
g^{\prime}=\left(D_{x} g\right) g
$$

where $D_{x} g$ is the Jacobian of $g$. Indeed, $g$ depends implicitly on $t$ through $x$, and hence the chain rule yields $g^{\prime}=\left(D_{x} g\right) x^{\prime}$; the result now follows from (2.1). Together, (2.1) and (2.2) describe the evolution of the pair $(x, g)$ in the $2 N$-dimensional tangent bundle $T \mathbb{R}^{N}$.

We now assume the dynamical system (2.1) to have a smooth, invariant manifold $\mathcal{M}$ of dimension $N_{2}<N$. For simplicity, we take $\mathcal{M}$ to be the graph of a function $h: K \rightarrow \mathbb{R}^{N_{1}}$, with $N_{1}=N-N_{2}$ and $K$ some subset of $\mathbb{R}^{N_{2}}$,

$$
\mathcal{M}=\left\{x \in \mathbb{R}^{N}: x=\left(x^{1}, x^{2}\right), x^{1}=h\left(x^{2}\right), x^{2} \in K\right\} .
$$

For any initial condition sufficiently close to $\mathcal{M}$, the solution of $(2.1)$ evolves on a fast time scale toward (a tubular neighborhood of) that manifold. Once there, it stays in that neighborhood and evolves on a 
much slower time scale, until it either reaches some attractor or exits through (a tubular neighborhood of) $\partial \mathcal{M}$. Because the motion near the manifold is slow relative to the motion away from it, $\mathcal{M}$ is also referred to as the "slow manifold."

The slow and fast directions depend on the distribution of the (generally complex and $x$-dependent) eigenvalues $\lambda_{1}, \ldots \lambda_{N}$ of $D_{x} g$. We assume that the eigenvalues separate into two disjoint groups,

$$
\Re\left(\lambda_{1}\right) \leq \cdots \leq \Re\left(\lambda_{N_{1}}\right) \leq \lambda_{f}<\lambda_{s} \leq \Re\left(\lambda_{N_{1}+1}\right) \leq \cdots \leq \Re\left(\lambda_{N}\right)<0,
$$

for some $\lambda_{f}$ and $\lambda_{s}$. The first $N_{1}$ eigenvalues constitute the "fast" part of the spectrum, and the corresponding eigenvectors span an $N_{1}$-dimensional fast eigenspace; the remaining $N_{2}=N-N_{1}$ eigenvalues constitute the "slow" part, and the corresponding eigenvectors span an $N_{2}$-dimensional slow eigenspace. No eigenvalue crosses the spectral gap, $\left\{\lambda \in \mathbb{C}: \lambda_{f} \leq \Re(\lambda) \leq \lambda_{s}\right\}$, over the time interval of interest.

The representation (2.3) induces a decomposition of $(2.1)$,

$$
\begin{aligned}
& x_{1}^{\prime}=g_{1}\left(x_{1}, x_{2}\right), \\
& x_{2}^{\prime}=g_{2}\left(x_{1}, x_{2}\right),
\end{aligned}
$$

and a corresponding decomposition of (2.2),

$$
\left(\begin{array}{l}
g_{1} \\
g_{2}
\end{array}\right)^{\prime}=\left(\begin{array}{ll}
D_{1} g_{1} & D_{2} g_{1} \\
D_{1} g_{2} & D_{2} g_{2}
\end{array}\right)\left(\begin{array}{l}
g_{1} \\
g_{2}
\end{array}\right) \text {. }
$$

Since $x_{1}=h\left(x_{2}\right)$ on $\mathcal{M}$, the system (2.5) reduces to a single ODE for the slow variable $x_{2}$ on $\mathcal{M}$,

$$
x_{2}^{\prime}=g_{2}\left(h\left(x_{2}\right), x_{2}\right) \quad \text { on } \mathcal{M} .
$$

This reduced equation is easier to solve, since it is non-stiff and the dimensionality $N_{2}$ of the slow subspace is less than $N$. Reduction methods are designed to find the function $h$. A large class of such methods revolves around the invariance equation, which is obtained by differentiating the identity $x_{1}=h\left(x_{2}\right)$ with respect to $t$,

$$
g_{1}-(D h) g_{2}=0 \quad \text { on } \mathcal{M} .
$$

Other identities can be derived from the invariance equation; for example, differentiating both members once with respect to $x_{2}$, we obtain the identity

$$
D_{2} g_{1}+\left(D_{1} g_{1}\right) D h-(D h)\left(D_{2} g_{2}+\left(D_{1} g_{2}\right) D h\right)-\left(D^{2} h\right) g_{2}=0 \quad \text { on } \mathcal{M} .
$$

Since $\mathcal{M}$ is invariant, the vector field on it lies entirely in the slow subspace, namely, the tangent space of $\mathcal{M}$ : for all $x \in \mathcal{M}, g(x) \in T_{x} \mathcal{M}$. If $\mathcal{M}$ is nonlinear, that slow subspace is not identical to the slow eigenspace of the Jacobian, and a local, linear analysis does not suffice to identify the function $h$. This discrepancy between slow eigenspace and slow subspace is easily traced back to representation (2.5) and its derivative (2.6). There, "fast" and "slow" vector field components are coupled and, consequently, fast and slow directions become entangled as the solution evolves along a trajectory. These directions can be separated, if we step away from the standard basis of unit vectors $\left\{e_{i}\right\}_{i=1}^{N}$ associated with the standard coordinatization of the state space $\mathbb{R}^{N}$. Instead, we will look for point-wise defined bases that reflect the local fast-slow structure of the vector field.

\section{Dynamics in the Tangent Bundle}

As mentioned, the ODEs (2.1)-(2.2) constitute the evolution laws in the tangent bundle for the problem at hand. In this section, we reformulate $(2.2)$ as a dynamic equation for the coefficients of the vector field $g$ in a local coordinate system.

Let $A$ be a nonsingular matrix whose entries may - and generally do-depend on $x$, and let $B$ denote its point-wise inverse. At each point $x \in \mathbb{R}^{N}$, the columns of $A(x)$ form a local basis for $T_{x} \mathbb{R}^{N}$ and, since 
$B_{i} A_{j}=\delta_{i, j}$, the rows of $B(x)$ form its dual basis. It follows that the vector field $g$ can be expressed in this local basis,

$$
g=A f, \quad \text { where } f=B g .
$$

The $x$-dependent components $f_{1}, \ldots, f_{N}$ of $f$ are the amplitudes of $g$ with respect to the basis $A$.

It is natural to derive laws dictating the temporal evolution of these amplitudes, as we did in (2.2) for the components relative to the standard basis. First, the relation $f=B g$ entails that $f^{\prime}=B g^{\prime}+B^{\prime} g$, with $g^{\prime}=\left(D_{x} g\right) g=\left(D_{x} g\right) A f$ by (2.2) and (3.1). Furthermore, $B g=B^{\prime} A f=-B A^{\prime} f$, where we have used the identity $B A=I$. Therefore, $f$ satisfies the ODE

$$
f^{\prime}=\Lambda f
$$

where $\Lambda$ is the operator

$$
\Lambda=B\left[\left(D_{x} g\right) A-A^{\prime}\right]
$$

Since $A^{\prime}=\left(D_{x} A\right) x^{\prime}=\left(D_{x} A\right) g$, that operator involves a Lie bracket [4,31] taken column-wise,

$$
\Lambda_{i j}=B_{i}\left[\left(D_{x} g\right) A_{j}-\left(D_{x} A_{j}\right) g\right]=B_{i}\left[A_{j}, g\right] \quad \text { or, compactly, } \Lambda=B[A, g] .
$$

Here, $B_{i}$ and $A_{j}$ denote the $i$-th row of $B$ and $j$-th column of $A$, respectively. Note that $\Lambda$ depends not only on $x$ but also on $g$ and, therefore, on $f$. Despite its appearance, then, (3.2) is a nonlinear ODE for the amplitudes $f$. In the special case where $A$ is the standard basis in $\mathbb{R}^{N}$, the expression (3.4) reduces to $\Lambda=D_{x} g$; cf. $(2.2)$.

\section{Invariance of $f$ and $\Lambda$}

In this section, we show that both the amplitudes $f$ and the operator $\Lambda$ are invariant under coordinate changes in $\mathbb{R}^{N}$. In other words, given a basis $A(x)$ for $T_{x} \mathbb{R}^{N}$ at each $x \in \mathbb{R}^{N}$, the amplitudes $f$ and the generator $\Lambda$ of their dynamics are well-defined irrespectively of the coordinates used in the state space.

We start by letting $\bar{x} \in \mathbb{R}^{N}$ be a new, curvilinear in general, $C^{\infty}$ set of coordinates replacing the original coordinates $x$. At each point $x \in \mathbb{R}^{N}$, this new coordinate set induces a basis for $T_{x} \mathbb{R}^{N}$ composed of the columns of $D_{\bar{x}} x$ and its dual composed of the rows of $D_{x} \bar{x}$ [38]. We write $\bar{A}$ and $\bar{B}$ for the given local bases expressed in these new coordinates and $\bar{g}$ for the vector field components with respect to $\bar{A}$,

$$
\bar{A}=\left(D_{x} \bar{x}\right) A, \quad \bar{B}=B\left(D_{\bar{x}} x\right), \quad \bar{g}=\left(D_{x} \bar{x}\right) g .
$$

The invariance of $f$ follows from a straightforward calculation relying on the identity $\left(D_{\bar{x}} x\right)\left(D_{x} \bar{x}\right)=I$,

$$
\bar{f}=\bar{B} \bar{g}=B\left(D_{\bar{x}} x\right)\left(D_{x} \bar{x}\right) g=B g=f .
$$

In essence, this calculation formalizes the observation that each amplitude is a rank-zero tensor (scalar) as the result of the action of a co-vector on a vector.

In a similar fashion, the fact that $\Lambda$ is invariant follows directly from the geometric characterization of $\Lambda$ as a Lie bracket. Indeed, $\left[A_{j}, g\right]$ is a vector field - that is, it transforms as such under coordinate changes [31] - and hence $B_{i}\left[A_{j}, g\right]$ is a scalar by the same token. Here also, we can arrive at that result through a direct calculation. According to (3.4), $\Lambda=B\left[\left(D_{x} g\right) A-\left(D_{x} A\right) g\right]=B[A, g]$. It follows from (4.1) that the expression for the operator in the new coordinates is

$$
\begin{aligned}
\bar{\Lambda} & =\bar{B}[\bar{A}, \bar{g}]=\bar{B}\left[\left(D_{\bar{x}} \bar{g}\right) \bar{A}-\left(D_{\bar{x}} \bar{A}\right) \bar{g}\right] \\
& =B\left(D_{\bar{x}} x\right)\left[\left(D_{\bar{x}}\left(\left(D_{x} \bar{x}\right) g\right)\right)\left(D_{x} \bar{x}\right) A-\left(\left(D_{\bar{x}}\left(D_{x} \bar{x}\right) A\right)\right)\left(D_{x} \bar{x}\right) g\right] .
\end{aligned}
$$

This expression can be simplified. First, we have the elementary identities $D_{\bar{x} x} \bar{x}=0$ and $D_{x} I=0$, as well as that $\left(D_{\bar{x}} x\right)\left(D_{x} \bar{x}\right)=I$. Second, $\left(D_{\bar{x}} g\right)\left(D_{x} \bar{x}\right)=D_{x} g$ and $\left(D_{\bar{x}} A\right)\left(D_{x} \bar{x}\right)=D_{x} A$, by the chain rule. Thus,

$$
\tilde{\Lambda}=B\left(D_{\bar{x}} x\right)\left(D_{x} \bar{x}\right)\left[\left(D_{\bar{x}} g\right)\left(D_{x} \bar{x}\right) A-\left(D_{\bar{x}} A\right)\left(D_{x} \bar{x}\right) g\right]=B[A, g]=\Lambda,
$$

which establishes the invariance of $\Lambda$. 


\section{Fast-Slow Decomposition}

At each $x \in \mathbb{R}^{N}$, the partition (2.4) of the spectrum of $D_{x} g$ induces a local decomposition of the tangent space $T_{x} \mathbb{R}^{N}$ into a fast eigenspace of dimension $N_{1}$ and a slow eigenspace of dimension $N_{2}$. This decomposition in turn induces a partition of $A$ into two column blocks, of size $N \times N_{1}$ and $N \times N_{2}$, and of $B$ into two row blocks, of size $N_{1} \times N$ and $N_{2} \times N$,

$$
A=\left(\begin{array}{ll}
A_{1} & A_{2}
\end{array}\right), \quad B=\left(\begin{array}{l}
B_{1} \\
B_{2}
\end{array}\right) .
$$

Note the identities $B_{1} A_{1}=I, B_{1} A_{2}=0, B_{2} A_{1}=0$, and $B_{2} A_{2}=I$. Then,

$$
f=\left(\begin{array}{l}
f_{1} \\
f_{2}
\end{array}\right)=\left(\begin{array}{l}
B_{1} g \\
B_{2} g
\end{array}\right)
$$

The governing equation (3.2) for the vector of amplitudes $f$ becomes a coupled system of (nonlinear) ODEs for $f_{1}$ and $f_{2}$,

$$
\left(\begin{array}{l}
f_{1} \\
f_{2}
\end{array}\right)^{\prime}=\left(\begin{array}{ll}
\Lambda_{11} & \Lambda_{12} \\
\Lambda_{21} & \Lambda_{22}
\end{array}\right)\left(\begin{array}{l}
f_{1} \\
f_{2}
\end{array}\right)
$$

where the elements $\Lambda_{i j}=B_{i}\left[A_{j}, g\right]$ are meant block-wise, for $i, j=1,2$. The equation (5.3) is central to the CSP method.

\section{Characterization of the Slow Manifold}

The slow manifold is the locus of points where the component of the vector field lying in the fast subspace is zero, so a first characterization of $\mathcal{M}$ is $f_{1}=0$. In terms of the original vector field components collected in $g$,

$$
\mathcal{M} \approx\left\{x \in \mathbb{R}^{N}: B_{1} g(x)=0\right\} .
$$

The accuracy of this characterization is contingent on that with which the rows of $B_{1}$ span the orthogonal complement of the slow subspace. Since these, in turn, are orthogonal to the columns of $A_{2}$, it is the quality with which the latter spans the slow subspace that determines the quality of (6.1).

To derive a second characterization, we use the invariance of $\Lambda$ under coordinate changes. Defining new local bases in $T_{x} \mathbb{R}^{N}$, in terms of near-identity transformations of $A$ and $B$, sends $A$ to $\hat{A}$ and $B$ to $\hat{B}$ via

$$
\hat{A}=A(I-U), \quad \hat{B}=(I+U) B, \quad \text { where } \quad U=\left(\begin{array}{cc}
0 & U_{12} \\
0 & 0
\end{array}\right) .
$$

The nonzero block $U_{12}$ depends on the slow variable, $U_{12} \equiv U_{12}\left(x_{2}\right)$, but is as yet undetermined. The block representations of $\hat{A}$ and $\hat{B}$ are

$$
\hat{A}=\left(\begin{array}{ll}
A_{1} A_{2}-A_{1} U_{12}
\end{array}\right), \quad \hat{B}=\left(\begin{array}{c}
B_{1}+U_{12} B_{2} \\
B_{2}
\end{array}\right),
$$

so the transformation leaves invariant the basis of the fast subspace (the columns of $A_{1}$ ) but changes that of the slow subspace (the columns of $A_{2}$ ). Note that $\hat{B}$ indeed inverts $\hat{A}$, as $(I-U)^{-1}=I+U$.

After changing vector bases, the condition (6.1) for the slow manifold becomes

$$
\hat{f}_{1}=\hat{B}_{1} g=\left(B_{1}+U_{12} B_{2}\right) g=0 .
$$

In the special case $A=I, B=I$, this condition reduces to

$$
g_{1}+U_{12} g_{2}=0
$$


Comparing this condition with the invariance equation (2.8), we see that the choice

$$
U_{12}=-D h
$$

reduces (6.5) to the invariance equation. The same conclusion holds in the general case if $A$ is not necessarily the identity. Hence, (6.6) is in a sense the ideal choice for $U_{12}$, as it recaptures the invariance equation and thus enables us to recover the slow manifold. In practice, the function $h$ is unknown and $U_{12}$ must be "constructed" in terms of $A$ and $\Lambda$.

To that effect, we compute that operator in the new coordinate system, starting from the expression $\hat{\Lambda}=\hat{B}\left[\left(D_{x} g\right) \hat{A}-(D \hat{A}) g\right]$. Since

$$
(I-U)^{\prime}=-U^{\prime}=-\left(D_{x} U\right) g=\left(\begin{array}{cc}
0 & -\left(D U_{12}\right) g_{2} \\
0 & 0
\end{array}\right),
$$

we see that

$$
\hat{\Lambda}=(I+U) B\left[\left(D_{x} g\right) A(I-U)-A^{\prime}(I-U)+A U^{\prime}\right]=(I+U) \Lambda(I-U)+U^{\prime} .
$$

The components of $\hat{\Lambda}$ are

$$
\begin{aligned}
& \hat{\Lambda}_{11}=\Lambda_{11}+U_{12} \Lambda_{21}, \\
& \hat{\Lambda}_{12}=\Lambda_{12}-\Lambda_{11} U_{12}+U_{12}\left(\Lambda_{22}-\Lambda_{21} U_{12}\right)+\left(D U_{12}\right) g_{2}, \\
& \hat{\Lambda}_{21}=\Lambda_{21}, \\
& \hat{\Lambda}_{22}=\Lambda_{22}-\Lambda_{21} U_{12} .
\end{aligned}
$$

Consider, in particular, the expression for $\hat{\Lambda}_{12}$. In the special case $A=B=I$, we have $\Lambda=D_{x} g$, so $\Lambda_{i j}=D_{j} g_{i}$, and the expression for $\hat{\Lambda}_{12}$ becomes

$$
\hat{\Lambda}_{12}=D_{2} g_{1}-\left(D_{1} g_{1}\right) U_{12}+U_{12}\left(D_{2} g_{2}-\left(D_{1} g_{2}\right) U_{12}\right)+\left(D U_{12}\right) g_{2} .
$$

If $U_{12}=-D h$, this expression is identical with the expression in the left member of $(2.9)$, which is zero on $\mathcal{M}$. Hence, $\hat{\Lambda}_{12}=0$ on $\mathcal{M}$ or, in other words, the evolution of the fast amplitudes decouples from that of the slow ones so that $f_{1}=0$ becomes an invariant set. But $\Lambda$ is invariant under coordinate transformations, so it must be the case that $\Lambda_{12}=0$ on $\mathcal{M}$. The same is true if one retains the general basis $B$, represents the slow manifold in terms of this basis, and then evaluates $\hat{\Lambda}_{12}$. Thus, a second characterization of the slow manifold is

$$
\mathcal{M}=\left\{x \in \mathbb{R}^{N}: \Lambda_{12}(x)=0\right\} .
$$

As noted before, however, the function $h$ is in general unknown, so the choice $U_{12}=-D h$ may be ideal, but it is generally unavailable.

The function $h$ and, therefore, the slow manifold $\mathcal{M}$ can be found by successive approximation through the (known) components of $\Lambda$. The one-step CSP method is an iterative process to achieve the identity $\Lambda_{12}=0$ by successive updates of the bases $A$ and $B$. The full CSP method goes one step further and aims to find bases $A$ and $B$ where $\Lambda$ reduces to block-diagonal form, $\Lambda_{12}=0$ and $\Lambda_{21}=0$. In these local coordinates, the fast and slow amplitudes of the vector field are completely decoupled.

\section{One-step CSP Method}

This iterative method starts with an arbitrary local basis, whose vectors are identified with the columns of the matrix $A^{(0)}$, and its dual basis, whose vectors are identified with the rows of the matrix $B^{(0)}$. After $n$ updates $(n=0,1, \ldots)$, the iterative procedure has generated a local basis represented by the columns 
of the matrix $A^{(n)}$ and a dual basis represented by the rows of the matrix $B^{(n)}$, with $B^{(n)} A^{(n)}=I$. The vector of amplitudes of the vector field $g$ relative to the basis $A^{(n)}$ is $f^{(n)}=B^{(n)} g$, and the dynamics of $f^{(n)}$ are generated by $\Lambda^{(n)}=B^{(n)}\left[A^{(n)}, g\right]$. The CSP manifold of order $n, \mathcal{M}^{(n)}$, is defined as the zero-level set of $f_{1}^{(n)}$,

$$
f_{1}^{(n)}(x)=B_{1}^{(n)} g(x)=0,
$$

and identified with the graph of a function $h^{(n)}$,

$$
\mathcal{M}^{(n)}=\left\{x \in \mathbb{R}^{N}: x=\left(x_{1}, x_{2}\right), x_{1}=h^{(n)}\left(x_{2}\right), x_{2} \in K \subset \mathbb{R}^{N_{2}}\right\} .
$$

The iteration uses a near-identity transformation, as in (6.2), which sends $A^{(n)}$ into $A^{(n+1)}=A^{(n)}(I-$ $\left.U^{(n)}\right)$ and $B^{(n)}$ into $B^{(n+1)}=\left(I+U^{(n)}\right) B^{(n)}$. The dynamics of $f^{(n+1)}=B^{(n+1)} g$ are generated by $\Lambda^{(n+1)}=B^{(n+1)}\left[A^{(n+1)}, g\right]$, with

$$
\Lambda_{12}^{(n+1)}=\Lambda_{12}^{(n)}-\Lambda_{11}^{(n)} U_{12}^{(n)}+U_{12}^{(n)}\left(\Lambda_{22}^{(n)}-\Lambda_{21}^{(n)} U_{12}^{(n)}\right)+\left(D U_{12}^{(n)}\right) g_{2} ;
$$

cf. (6.8). Recall that $U_{12}^{(n)}$ depends on $x_{2}$ but is still undetermined. In the CSP method, $U_{12}^{(n)}$ is taken as

$$
U_{12}^{(n)}=\left(\Lambda_{11}^{(n)}\right)^{-1} \Lambda_{12}^{(n)}
$$

This choice eliminates the first two terms in the expression for $\Lambda_{12}^{(n+1)}$ and leaves the expression

$$
\Lambda_{12}^{(n+1)}=U_{12}^{(n)}\left(\Lambda_{22}^{(n)}-\Lambda_{21}^{(n)} U_{12}^{(n)}\right)+\left(D U_{12}^{(n)}\right) g_{2} .
$$

Remark. In fast-slow systems, the expression (7.4) is of higher order in the small parameter $\varepsilon$, in a neighborhood of $\mathcal{M}$, so the definition (7.3) brings us closer to the identity $\Lambda_{12}=0$ as $n$ increases. For each successive $n$, the manifold $\mathcal{M}^{(n)}$ is known to be an improved approximation to $\mathcal{M}[36]$.

\subsection{Iterations and Coordinate Changes Commute}

We recall from Section 4 that the amplitudes $f$ and operator $\Lambda$ are independent of the coordinate system $x$ used to coordinatize $\mathbb{R}^{N}$. We now show that the operations of changing coordinates in $\mathbb{R}^{N}$ and performing an iteration of the one-step CSP method commute. The commutativity is expressed in the following diagram, where $A^{(n)}, B^{(n)}, f^{(n)}$, and $\Lambda^{(n)}$ (top left) are the values of $A, B, f$, and $\Lambda$ after $n$ iterations.

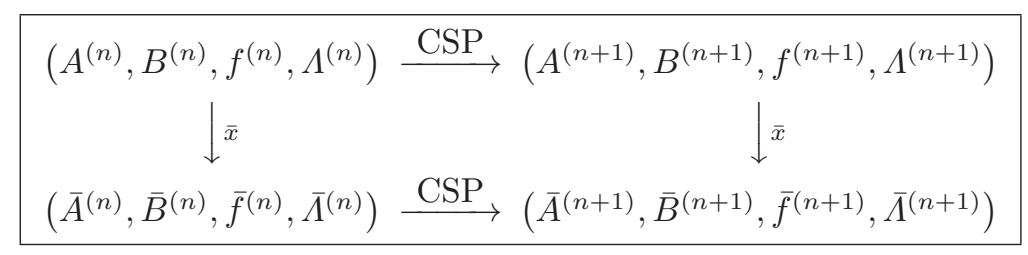

Starting from ( $n$-th iterate) quantities expressed in the coordinate system $x$ (top left) and performing a CSP iterate (top arrow to top right) yields the same result as first passing to new coordinates (down arrow to bottom left), then performing a CSP iteration (bottom arrow to bottom right), and finally returning to the original coordinates (against the arrow to the top right). We prove this by computing, first, the result of following the down arrow marked ' $\bar{x}$ ' to bottom left and subsequently the right arrow marked 'CSP' to bottom right; and second, the result of following the right arrow marked 'CSP' to top right and subsequently following the down arrow marked ' $\bar{x}$ ' to the bottom left.

For the first computation, let $\bar{x}: \mathbb{R}^{N} \rightarrow \mathbb{R}^{N}$ be a $C^{\infty}$ change of coordinates inducing, at every point $x_{0} \in \mathbb{R}^{N}$, the new local basis $D_{\bar{x}} x\left(x_{0}\right)$ and its corresponding dual $D_{x} \bar{x}\left(x_{0}\right)$. In this basis, $g$ and $A^{(n)}$ are 
represented by $\bar{g}$ and $\bar{A}^{(n)}$; in its dual, $B^{(n)}$ is represented by $\bar{B}^{(n)}$. The expressions are as in (4.1),

$$
\begin{aligned}
g & =\left(D_{\bar{x}} x\right) \bar{g}, & & \text { where } \bar{g}=\left(D_{x} \bar{x}\right) g, \\
A^{(n)} & =\left(D_{\bar{x}} x\right) \bar{A}^{(n)}, & & \text { where } \bar{A}^{(n)}=\left(D_{x} \bar{x}\right) A^{(n)}, \\
B^{(n)} & =\bar{B}^{(n)}\left(D_{x} \bar{x}\right), & & \text { where } \bar{B}^{(n)}=B^{(n)}\left(D_{\bar{x}} x\right) .
\end{aligned}
$$

Similarly, $f^{(n)}$ becomes $\bar{f}^{(n)}$ and $\Lambda^{(n)}$ becomes $\bar{\Lambda}^{(n)}$; by the invariance results in Section 4 ,

$$
f^{(n)}=\bar{f}^{(n)} \quad \text { and } \quad \Lambda^{(n)}=\bar{\Lambda}^{(n)} .
$$

The change of coordinates is followed by a CSP iteration to update the bases,

$$
\begin{aligned}
& \bar{A}^{(n+1)}=\bar{A}^{(n)}\left(I-\bar{U}^{(n)}\right), \\
& \bar{B}^{(n+1)}=\left(I+\bar{U}^{(n)}\right) B^{(n)},
\end{aligned}
$$

where

$$
\bar{U}_{12}^{(n)}=\left(\bar{\Lambda}_{11}^{(n)}\right)^{-1} \bar{\Lambda}_{12}^{(n)}=\left(\Lambda_{11}^{(n)}\right)^{-1} \Lambda_{12}^{(n)}=U_{12}^{(n)} .
$$

With this new basis, we can generate $\bar{f}^{(n+1)}$ and $\bar{\Lambda}^{(n+1)}$ and thus complete the computation.

For the second computation, we first update the bases $A^{(n)}$ and $B^{(n)}$,

$$
\begin{aligned}
& A^{(n+1)}=A^{(n)}\left(I-U^{(n)}\right), \\
& B^{(n+1)}=\left(I+U^{(n)}\right) B^{(n)} .
\end{aligned}
$$

The subsequent coordinate change gives the identities

$$
\begin{aligned}
& \left(D_{\bar{x}} x\right) \overline{A^{(n+1)}}=\left(D_{\bar{x}} x\right) \overline{A^{(n)}}\left(I-U^{(n)}\right), \\
& \overline{B^{(n+1)}}\left(D_{x} \bar{x}\right)=\left(I+U^{(n)}\right) \overline{B^{(n)}}\left(D_{x} \bar{x}\right)
\end{aligned}
$$

cf. (7.6) and (7.7). By left multiplying the first equation by $D_{x} \bar{x}$ and right multiplying the second equation by $D_{\bar{x}} x$, we obtain the expressions

$$
\begin{aligned}
& \overline{A^{(n+1)}}=\overline{A^{(n)}}\left(I-U^{(n)}\right), \\
& \overline{B^{(n+1)}}=\left(I+U^{(n)}\right) \overline{B^{(n)}} .
\end{aligned}
$$

With this new basis we can obtain $\bar{f}_{(n+1)}$ and $\bar{\Lambda}^{(n+1)}$ and complete the computation.

The equivalence of the expressions (7.9) and (7.11), and thus the commutativity of the coordinate change and the CSP iteration, follows from (7.10). The proof of the invariance of $\Lambda^{(n)}$ is similar to the proof given for $\Lambda$ in Section 4 .

\subsection{Generating the Slow Manifold}

We now show how the implementation of the one-step CSP method generates successive approximations to the function $h$.

The method is normally started from the standard basis, $A^{(0)}=B^{(0)}=I$, where $\Lambda^{(0)}=D_{x} g$. After $n$ applications of the update procedure (6.2), the method generates a basis $A^{(n)}$ and its dual $B^{(n)}$,

$$
A^{(n)}=\left(\begin{array}{cc}
I_{1}-P^{(n-1)} \\
0 & I_{2}
\end{array}\right), \quad B^{(n)}=\left(\begin{array}{cc}
I_{1} & P^{(n-1)} \\
0 & I_{2}
\end{array}\right),
$$


where

$$
P^{(-1)}=0 ; \quad P^{(n-1)}=\sum_{i=0}^{n-1} U_{12}^{(i)}, \quad n=1,2, \ldots
$$

The manifold $\mathcal{M}^{(n)}$ is determined by the equation $f_{1}^{(n)}=B_{1}^{(n)} g=0$, which shares the same form with the invariance equation,

$$
g_{1}+P^{(n-1)} g_{2}=0 \quad \text { on } \mathcal{M}^{(n)}
$$

Now, $\mathcal{M}^{(n)}$ is the graph of a function $h^{(n)}: K \rightarrow \mathbb{R}^{N_{1}}$,

$$
\mathcal{M}^{(n)}=\left\{x=\left(x_{1}, x_{2}\right): x_{1}=h^{(n)}\left(x_{2}\right), x_{2} \in K \subset \mathbb{R}^{N_{2}}\right\} .
$$

Hence, differentiation of (7.14) with respect to $x_{2}$ yields the additional identity

$$
D_{2} g_{1}+\left(D_{1} g_{1}\right)\left(D h^{(n)}\right)+P^{(n-1)}\left(D_{2} g_{2}+\left(D_{1} g_{2}\right) D h^{(n)}\right)+\left(D P^{(n-1)}\right) g_{2}=0 \quad \text { on } \mathcal{M}^{(n)}
$$

Next, we consider the elements $\Lambda_{11}^{(n)}$ and $\Lambda_{12}^{(n)}$ of $\Lambda^{(n)}=B^{(n)}\left[A^{(n)}, g\right]$,

$$
\begin{aligned}
& \Lambda_{11}^{(n)}=D_{1} g_{1}+P^{(n-1)}\left(D_{1} g_{2}\right), \\
& \Lambda_{12}^{(n)}=D_{2} g_{1}-\left(D_{1} g_{1}\right) P^{(n-1)}+P^{(n-1)}\left(D_{2} g_{2}-\left(D_{1} g_{2}\right) P^{(n-1)}\right)+\left(D P^{(n-1)}\right) g_{2} .
\end{aligned}
$$

We evaluate these expressions on $\mathcal{M}^{(n)}$ and use the values in the determining equation for $U_{12}^{(n)}$, namely $\Lambda_{12}^{(n)}-\Lambda_{11}^{(n)} U_{12}^{(n)}=0$,

$$
\begin{aligned}
D_{2} g_{1} & -\left(D_{1} g_{1}\right) P^{(n-1)}+P^{(n-1)}\left(D_{2} g_{2}-\left(D_{1} g_{2}\right) P^{(n-1)}\right)+\left(D P^{(n-1)}\right) g_{2} \\
& -\left(D_{1} g_{1}+P^{(n-1)}\left(D_{1} g_{2}\right)\right) U_{12}^{(n)}=0 \quad \text { on } \mathcal{M}^{(n)} .
\end{aligned}
$$

The equations (7.14) and (7.16) are identical if

$$
U_{12}^{(n)}=-P^{(n-1)}-D h^{(n)} .
$$

Consequently, $P^{(n)}=P^{(n-1)}+U_{12}^{(n)}=-D h^{(n)}$, and therefore

$$
U_{12}^{(n)}=-D\left(h^{(n)}-h^{(n-1)}\right), \quad n=0,1, \ldots .
$$

This illustrates how each CSP iterate defines a successive approximation of the slow manifold, as was proven in [36]. Moreover, it is of interest to use this approach as the basis for a contraction mapping-type argument to prove CSP validity for general systems with a spectral gap.

\section{CSP and Fast-Slow Systems}

The commutativity of a coordinate change and an update in the one-step CSP method implies that, for the purpose of analyzing the CSP method, we may express the system in coordinates that are most suitable for the dynamics. In this section, we will analyze the one-step CSP method for fast-slow systems, where the separation of time scales is expressed by a positive but arbitrarily small parameter $\varepsilon$.

Standard asymptotic theory guarantees that, under appropriate conditions on the vector field $g$, there exists a slow manifold $\mathcal{M}_{\varepsilon}$ that is invariant under the dynamics of the system. All nearby solutions relax exponentially fast to $\mathcal{M}_{\varepsilon}$, and their long-term evolution is determined by an associated solution on the slow manifold itself $[5,18]$. 
The Fenichel theory leads to the normal form for the vector field in the neighborhood of $\mathcal{M}_{\varepsilon}$,

$$
\begin{aligned}
b^{\prime} & =\Gamma\left(b, x_{2}, \varepsilon\right) b, \\
x_{2}^{\prime} & =\varepsilon m_{1}\left(x_{2}, \varepsilon\right)+m_{2}\left(b, x_{2}, \varepsilon\right) b,
\end{aligned}
$$

where $b: t \mapsto b(t) \in \mathbb{R}^{N_{1}}$ and $x_{2}: t \mapsto x_{2}(t) \in \mathbb{R}^{N_{2}}$. The $N_{1}$ eigenvalues of $\Gamma\left(0,0, x_{2}, 0\right)$ all have negative real part, and $\Lambda, \Gamma, m_{1}$ and $m_{2}$ and their derivatives are all smooth in $\varepsilon$. The Fenichel normal form (8.1) fits in the general framework of (2.1),

$$
x=\left(\begin{array}{c}
b \\
x_{2}
\end{array}\right), \quad g=\left(\begin{array}{c}
\Gamma\left(b, x_{2}, \varepsilon\right) b \\
\varepsilon m_{1}\left(x_{2}, \varepsilon\right)+m_{2}\left(b, x_{2}, \varepsilon\right) b
\end{array}\right) .
$$

Hence,

$$
D_{x} g=\left(\begin{array}{cc}
\left(D_{b} \Gamma\right) b+\Gamma & \left(D_{2} \Gamma\right) b \\
\left(D_{b} m_{2}\right) b+m_{2} & \varepsilon D_{2} m_{1}+\left(D_{2} m_{2}\right) b
\end{array}\right) .
$$

In the standard basis, $A^{(0)}=B^{(0)}=I$, the amplitudes are

$$
\begin{aligned}
& f_{1}^{(0)}=B_{1}^{(0)} g=g_{1}, \\
& f_{2}^{(0)}=B_{2}^{(0)} g=g_{2},
\end{aligned}
$$

and $\Lambda^{(0)}=D_{x} g$, by (3.4). Therefore, application of the condition (6.4) yields the set

$$
\mathcal{M}^{(0)}=\{b=0\},
$$

since $\Gamma$ in non-degenerate in the neighborhood of $\mathcal{M}_{\varepsilon}$. In general, $\mathcal{M}^{(0)}$ is only an approximation of the slow invariant manifold. In this case, since we are working with the Fenichel normal form, $\mathcal{M}^{(0)}$ is exactly the slow invariant manifold $\mathcal{M}_{\varepsilon}$.

We now show that the Fenichel normal form and the set $\{b=0\}$ are invariants of the iteration. With $\left.\left(\Lambda_{11}^{(0)}\right)^{-1}\right|_{\mathcal{M}^{(0)}}=\left(\Gamma\left(0, x_{2}, \varepsilon\right)\right)^{-1}$ and $\left.\Lambda_{12}^{(0)}\right|_{\mathcal{M}^{(0)}}=0$, we obtain

$$
U_{12}^{(0)}=\left(\Lambda_{11}^{(0)}\right)^{-1} \Lambda_{12}^{(0)}=0 \quad \text { on } \mathcal{M}^{(0)} .
$$

It follows immediately that $B^{(1)}=B^{(0)}$ and

$$
\begin{aligned}
& f_{1}^{(1)}=B_{1}^{(1)} g=B_{1}^{(0)} g=g_{1}, \\
& f_{2}^{(1)}=B_{2}^{(1)} g=B_{2}^{(0)} g=g_{2},
\end{aligned}
$$

which shows that the Fenichel normal form is invariant under iteration in the one-step CSP method. Moreover, application of the condition (6.4) yields $\mathcal{M}^{(1)}=\{b=0\}$, so $\mathcal{M}^{(1)}$ is the same as $\mathcal{M}^{(0)}$. Therefore, $\mathcal{M}_{\varepsilon}$ is also invariant under iteration of the one-step CSP method.

This proof of the validity of the CSP method is concise and modular, and it sheds useful new insights into the method.

\section{Example of a Fast-Slow System}

The following planar example of a fast-slow system is due to Davis and Skodje [3]:

$$
\begin{aligned}
& x_{1}^{\prime}=g_{1}\left(x_{1}, x_{2}, \varepsilon\right)=-x_{1}+\frac{x_{2}}{1+x_{2}}-\varepsilon \frac{x_{2}}{\left(1+x_{2}\right)^{2}}, \\
& x_{2}^{\prime}=g_{2}\left(x_{1}, x_{2}, \varepsilon\right)=-\varepsilon x_{2} .
\end{aligned}
$$


The system is constructed in such a way that it has an invariant manifold,

$$
\mathcal{M}=\left\{x=\left(x_{1}, x_{2}\right): x_{1}=\frac{x_{2}}{1+x_{2}}\right\} .
$$

This manifold is independent of $\varepsilon$ and is also the slow manifold for the system if $\varepsilon=0$.

We apply the CSP method, starting with the standard basis. The amplitudes are

$$
f_{1}^{(0)}=B_{1}^{(0)} g=g_{1}, \quad f_{2}^{(0)}=B_{2}^{(0)} g=g_{2},
$$

and $\Lambda^{(0)}=D_{x} g$. Application of the condition (7.1) yields

$$
\mathcal{M}^{(0)}=\left\{x=\left(x_{1}, x_{2}\right): x_{1}=\frac{x_{2}}{1+x_{2}}-\varepsilon \frac{x_{2}}{\left(1+x_{2}\right)^{2}}\right\},
$$

which agrees to leading order with (9.2). For the first iteration, we use

$$
U_{12}^{(0)}=\left(\Lambda_{11}^{(0)}\right)^{-1} \Lambda_{12}^{(0)}=\frac{-1}{\left(1+x_{2}\right)^{2}}+\varepsilon \frac{1-x_{2}}{\left(1+x_{2}\right)^{3}} .
$$

After the iteration,

$$
f_{1}^{(1)}=B_{1}^{(1)} g=-x_{1}+\frac{x_{2}}{1+x_{2}}-\varepsilon^{2} \frac{x_{2}\left(1-x_{2}\right)}{\left(1+x_{2}\right)^{3}},
$$

and the new approximation to the slow manifold is

$$
\mathcal{M}^{(1)}=\left\{x=\left(x_{1}, x_{2}\right): x_{1}=\frac{x_{2}}{1+x_{2}}-\varepsilon^{2} \frac{x_{2}\left(1-x_{2}\right)}{\left(1+x_{2}\right)^{3}}\right\} .
$$

This is an improvement over $\mathcal{M}^{(0)}$; the remainder is now of second order in $\varepsilon$. In fact, each successive iteration pushes the remainder one order higher in $\varepsilon$.

Note that $\Lambda_{21}^{(n)}=0$ for all $n$, since $g_{2}$ is independent of $x_{1}$. It follows from (6.8) and (7.4) that

$$
\begin{aligned}
& \Lambda_{11}^{(1)}=\Lambda_{11}^{(0)}=-1, \\
& \Lambda_{12}^{(1)}=\varepsilon \frac{1-x_{2}}{\left(1+x_{2}\right)^{3}}-\varepsilon^{2} \frac{5-2 x_{2}-x_{2}^{2}}{\left(1+x_{2}\right)^{4}} .
\end{aligned}
$$

Hence,

$$
U_{12}^{(1)}=-\varepsilon\left(\frac{1-x_{2}}{\left(1+x_{2}\right)^{3}}\right)+\varepsilon^{2}\left(\frac{5-2 x_{2}-x_{2}^{2}}{\left(1+x_{2}\right)^{4}}\right) .
$$

The term of $\mathcal{O}(\varepsilon)$ cancels the same term in $(9.4)$, so $U_{12}^{(0)}+U_{12}^{(1)}=\frac{-1}{\left(1+x_{2}\right)^{2}}+\mathcal{O}\left(\varepsilon^{2}\right)$. This cancellation occurs at every iteration and order. Hence,

$$
\lim _{n \rightarrow \infty} \sum_{i=0}^{n} U_{12}^{(i)}=\frac{-1}{\left(1+x_{2}\right)^{2}},
$$

which illustrates the theory of Section 7.2.

Remark. In this example, we used the standard basis as the initial basis. As may be seen from (9.8), the optimal initial basis in $\mathbb{R}^{2}$ is

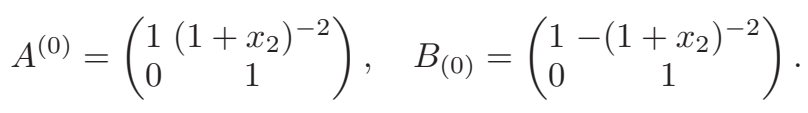

Then one immediately obtains the exact manifold, $\mathcal{M}^{(0)}=\mathcal{M}$, and no further iterations are needed. 


\section{Two-step CSP Method}

The two-step CSP method is designed to completely separate the dynamics of the fast and slow variables in (2.1). The separation is achieved by a reduction of the operator $\Lambda$ in (3.2) to block-diagonal form. Each step of the iterative method involves two transformations, one to change the basis of the slow subspace and another to change the basis of the fast subspace,

$$
\hat{A}=A(I-U)(I+L), \quad \hat{B}=(I+L)^{-1}(I-U)^{-1} B,
$$

where

$$
\begin{aligned}
& U=\left(\begin{array}{cc}
0 & U_{12} \\
0 & 0
\end{array}\right), \quad U_{12}=\left(\Lambda_{11}\right)^{-1} \Lambda_{12}, \\
& L=\left(\begin{array}{cc}
0 & 0 \\
L_{21} & 0
\end{array}\right), \quad L_{21}=\Lambda_{21}\left(\Lambda_{11}\right)^{-1} .
\end{aligned}
$$

The elements $U_{12}$ and $L_{21}$ are functions only of the slow variable $x_{2}$, and the matrices $U_{12}$ and $L_{21}$ are chosen so that the leading quantities in $\hat{\Lambda}_{12}$ and $\hat{\Lambda}_{21}$ vanish. Also, $U$ and $L$ are nilpotent, so $(I-U)^{-1}=I+U$ and $(I+L)^{-1}=I-L$.

A straightforward computation gives

$$
\hat{\Lambda}=(I-L)(I+U) \Lambda(I-U)(I+L)+(I-L) U^{\prime}(I+L)-L^{\prime} .
$$

The key component $\hat{\Lambda}_{12}$ is given by the expression

$$
\hat{\Lambda}_{12}=\Lambda_{12}-\Lambda_{11} U_{12}+U_{12}\left(\Lambda_{22}-\Lambda_{21} U_{12}\right)+\left(D U_{12}\right) g_{2},
$$

which is the same as the expression (6.9) for the one-step CSP method. Using the same arguments as before, we conclude that the slow manifold is characterized by the same condition (6.10),

$$
\mathcal{M}=\left\{x \in \mathbb{R}^{N}: \Lambda_{12}(x)=0\right\} .
$$

Commutativity of the operations of changing coordinates and performing an iteration of the two-step CSP method is established as for the one-step method. We recall from Section 7.1 the key observation that $\Lambda$ is independent of the coordinate system, which holds here as well. Using the same notation as in that section, we find that the first path in the commutative diagram leads to the expressions

$$
\begin{aligned}
& \bar{A}^{(n+1)}=\bar{A}^{(n)}\left(I-\bar{U}^{(n)}\right)\left(I+\bar{L}^{n}\right), \\
& \bar{B}^{(n+1)}=\left(I-\bar{L}^{(n)}\right)\left(I+\bar{U}^{(n)}\right) B^{(n)},
\end{aligned}
$$

while the second path leads to the expressions

$$
\begin{aligned}
& \overline{A^{(n+1)}}=\overline{A^{(n)}}\left(I-U^{(n)}\right)\left(I+L^{(n)}\right), \\
& \overline{B_{(n+1)}}=\left(I-L^{(n)}\right)\left(I+U^{(n)}\right) \overline{B_{(n)}} .
\end{aligned}
$$

The invariance property $\bar{\Lambda}^{(n)}=\Lambda^{(n)}$ implies also that $\bar{U}^{(n)}=U^{(n)}$ and $\bar{L}^{(n)}=L^{(n)}$, and hence (10.6) and (10.7) are identical. Hence, the same commutative diagram applies for the two-step CSP method, and an analysis of the method may be carried out in a coordinate system best attuned to the dynamics.

In particular, we work with the Fenichel normal form (8.1). Taking the initial basis to be the standard basis, we start with

$$
\begin{aligned}
& f_{1}^{(0)}=B_{1}^{(0)} g=g_{1}, \\
& f_{2}^{(0)}=B_{2}^{(0)} g=g_{2},
\end{aligned}
$$


and $\Lambda^{(0)}=D_{x} g$. Then,

$$
\mathcal{M}^{(0)}=\{b=0\} .
$$

Evaluating on $\mathcal{M}^{(0)}$ the matrices used to update the bases, we obtain

$$
\begin{aligned}
U_{12}^{(0)} & =\left(\Lambda_{11}^{(0)}\right)^{-1} \Lambda_{12}^{(0)}=0 \\
L_{21}^{(0)} & =\Lambda_{21}^{(0)}\left(\Lambda_{11}^{(0)}\right)^{-1}=m_{2}\left(0, x_{2}, \varepsilon\right)\left(\Gamma\left(0, x_{2}, \varepsilon\right)\right)^{-1} .
\end{aligned}
$$

The bases transform to

$$
\begin{aligned}
& A^{(1)}=A^{(0)}\left(I-U^{(0)}\right)\left(I+L^{(0)}\right)=\left(\begin{array}{cc}
I_{1} & 0 \\
m_{2}\left(0, x_{2}, \varepsilon\right)\left(\Gamma\left(0, x_{2}, \varepsilon\right)\right)^{-1} & I_{2}
\end{array}\right), \\
& B^{(1)}=\left(I-L^{(0)}\right)\left(I+U^{(0)}\right) B^{(0)}=\left(\begin{array}{cc}
I_{1} & 0 \\
-m_{2}\left(0, x_{2}, \varepsilon\right)\left(\Gamma\left(0, x_{2}, \varepsilon\right)\right)^{-1} & I_{2}
\end{array}\right) .
\end{aligned}
$$

Hence, the amplitudes become

$$
\begin{aligned}
& f_{1}^{(1)}=B_{1}^{(1)} g=g_{1}, \\
& f_{2}^{(1)}=B_{2}^{(1)} g=-m_{2}\left(0, x_{2}, \varepsilon\right)\left(\Gamma\left(0, x_{2}, \varepsilon\right)\right)^{-1} g_{1}+g_{2} .
\end{aligned}
$$

Thus, the condition $f_{1}^{(1)}=0$ yields

$$
\mathcal{M}^{(1)}=\{b=0\},
$$

and the slow manifold is again invariant. The second component of the vector field transforms to

$$
f_{2}^{(1)}=\varepsilon m_{1}\left(x_{2}, \varepsilon\right)+\tilde{m}_{2}\left(b, x_{2}, \varepsilon\right) b,
$$

where

$$
\tilde{m}_{2}\left(b, x_{2}, \varepsilon\right)=m_{2}\left(b, x_{2}, \varepsilon\right)-\left(m_{2}\left(0, x_{2}, \varepsilon\right)\left(\Gamma\left(0, x_{2}, \varepsilon\right)\right)^{-1}\right) \Gamma\left(b, x_{2}, \varepsilon\right) .
$$

This implies that the flow on the slow manifold is preserved under iteration of the full CSP method, but the dynamics on the fibers is altered.

Acknowledgements. The work of T.K. was supported in part by the Division of Mathematical Sciences of the National Science Foundation via grant NSF-1109587. The work of A.Z. was supported in part by the Division of Physical Sciences of the Netherlands Organisation for Scientific Research via grant 639.031.617.

\section{References}

[1] T.M.K. Coles, H.N Najm, Y.M. Marzouk. CSP simplification of chemical kinetic systems under uncertainty. In: Proc. Third IWMRRF. Corfu, Greece, April 27-29, 2011, 331-334.

[2] B.J. Debusschere, Y.M. Marzouk, H.N. Najm, B. Rhoads, D.A. Goussis, and M. Valorani. Computational sinular perturbation with non-parametric tabulation of slow manifolds for time integration of stiff chemical systems. Combust. Theor. Model., 16 (2012), 173-198.

[3] M.J. Davis, R.T. Skodje. Geometric investigation of low-dimensional manifolds in systems approaching equilibrium. J. Chem. Phys., 111 (1999), 859-874.

[4] B.A. Dubrovin, A.T. Fomenko, S.P. Novikov. Modern Geometry - Methods and Applications, vol. 2. Graduate Texts in Mathematics, 104. Springer-Verlag, New York, 1985.

[5] N. Fenichel. Geometric singular perturbation theory for ordinary differential equations. J. Diff. Eq., 31 (1979), 53-98.

[6] A.N. Gorban, I.V. Karlin. Method of Invariant Manifold for chemical kinetics. Chem. Eng. Sci., 58 (2003), $4751-4768$.

[7] A.N. Gorban, I.V. Karlin. Invariant Manifolds for Physical and Chemical Kinetics. Springer, Berlin, 2004.

[8] A.N. Gorban, I.V. Karlin. Hilbert's 6th Problem: Exact and Approximate Hydrodynamic Manifolds for Kinetic Equations. Bulletin Amer. Math. Soc., 51 (2014), 187-246.

[9] A.N. Gorban, I.V. Karlin, A.Yu. Zinovyev. Constructive methods for invariant manifolds for kinetic problems. Phys. Reports, 396 (2004), 197-403. 
[10] A.N. Gorban, N. Kazantzis, Y.G. Kevrekidis, H.C. Ottinger, and C. Theodoropoulos (eds.). Model Reduction and Coarse-Graining Approaches for Multiscale Phenomena. Springer, Berlin, 2006.

[11] D.A. Goussis, S.H. Lam. A study of homogeneous methanol oxidation kinetics using CSP. In: Proceedings of the Twenty-Fourth Symposium (International) on Combustion, The University of Sydney, Sydney, Australia, July 5-10, 1992. The Combustion Institute, Pittsburgh, 1992, 113-120.

[12] D. Goussis, U. Maas. Model reduction for combustion chemistry. In: Turbulent Combustion Modeling, Fluid Mechanics and Its Applications, vol. 95. Springer, (2011), 193-220.

[13] D. Goussis, H.N. Najm. Model reduction and physical understanding of slowly oscillating processes: the circadian cycle. Multiscale Model. Sim., 5 (2006), 1297-1332.

[14] D. Goussis, M. Valorani. An efficient iterative algorithm for the approximation of fast and slow dynamics of stiff systems. J. Comp. Phys., 214 (2006), 316-346.

[15] S. Gupta. High-Fidelity Simulation and Analysis of Ignition Regimes and Mixing Characteristics for Low Temperature Combustion Engine Applications. Ph.D. Thesis, U. Michigan, 2012.

[16] M. Hadjinicolaou, D.A. Goussis. Asymptotic solutions of stiff PDEs with the CSP method: The reaction diffusion equation. SIAM J. Sci. Comput., 20 (1999), 781-810.

[17] H. Hardin, A. Zagaris, K. Krab, H.W. Westerhoff. Simplified yet highly accurate enzyme kinetics for cases of low substrate concentrations. Fed. Eur. Biochem. Soc. J., 276 (2009), 5491-5506.

[18] C.K.R.T. Jones. Geometric singular perturbation theory. In: Dynamical Systems, Montecatini Terme, L. Arnold, Lecture Notes in Mathematics, 1609. Springer-Verlag, Berlin, 1994, 44-118.

[19] H.G. Kaper, T.J. Kaper. Asymptotic analysis of two reduction methods for systems of chemical kinetics. Physica D, 165 (2002), 66-93.

[20] P.D. Kourdis, A.G. Palasantza, D.A. Goussis. Algorithmic asymptotic analysis of the NF-kB signaling system. Comp. Math. Appl., 65 (2013), 1516-1534.

[21] P.D. Kourdis, R. Steuer, D.A. Goussis. Physical understanding of complex multiscale biochemical models via algorithmic simplification: Glycolysis in Saccharomyces cerevisiae. Physica D, 239 (2010), 1798-1817.

[22] S.H. Lam. Using CSP to understand complex chemical kinetics. Combust. Sci. Tech., 89 (1993), 375-404.

[23] S.H. Lam. Reduced chemistry-diffusion coupling. Combust. Sci. Tech., 179 (2007), 767-786.

[24] S.H. Lam, D.A. Goussis. Understanding complex chemical kinetics with computational singular perturbation. In: Proceedings of the Twenty-Second Symposium (International) on Combustion, The University of Washington, Seattle, Washington, August 14-19, 1988. The Combustion Institute, Pittsburgh, 1988, 931-941.

[25] S.H. Lam, D.A. Goussis. Conventional asymptotics and computational singular perturbation theory for simplified kinetics modeling. In: Reduced Kinetic Mechanisms and Asymptotic Approximations for Methane-Air Flames, M. Smooke (ed.). Lecture Notes in Physics, 384. Springer-Verlag, New York, 1991, Chapter 10.

[26] S.H. Lam, D.A. Goussis. The CSP method for simplifying kinetics. Internat. J. Chem. Kin., 26 (1994), 461-486.

[27] T. Lovas, E. Mastorakos, D.A. Goussis. Reduction of the RACM scheme using CSP in atmospheric chemistry modeling. J. Geophys. Res. - Atmos., 111 (2006), 1-16.

[28] T.F. Lu, Y.G. Ju, C.K. Law. Complex CSP for chemistry reduction and analysis. Combust. Flame, 126 (2001), 14451455.

[29] A. Massias, D. Diamantis, E. Mastorakos, D. Goussis. Global reduced mechanisms for methane and hydrogen combustion with nitric oxide formation constructed with CSP data. Combust. Theor. Model., 3 (1999), 233-257.

[30] M.K. Neophytou, D.A. Goussis, M. van Loon, E. Mastorakos. Reduced chemical mechanisms for atmospheric pollution using computational singular perturbation analysis. Atmos. Environ., 38 (2004), 3661-3673.

[31] P.J. Olver. Applications of Lie Groups to Differential Equations, Graduate Texts in Mathematics, vol. 107. SpringerVerlag, New York, 1986.

[32] M. Valorani, F. Creta, D.A. Goussis, H.N. Najm, J.C. Lee. Chemical kinetics mechanism simplification via CSP. Combust. Flame, 146 (2006), 29-51.

[33] M. Valorani, D.A. Goussis, F. Creta, H.N. Najm. Higher-order corrections in the approximation of inertial manifolds and the construction of simplified problems with the CSP method. J. Comp. Phys., 209 (2005), 754-786.

[34] M. Valorani, D.A. Goussis. Explicit time-scale splitting algorithm for stiff problems: auto-ignition of gaseous mixtures behind a steady shock. J. Comput. Phys., 169 (2001), 44-79.

[35] M. Valorani, H.M. Najm, D.A. Goussis. CSP analysis of a transient flame-vortex interaction: time scales and manifolds. Combust. Flame, 134 (2003), 35-53.

[36] A. Zagaris, H.G. Kaper, T.J. Kaper. Analysis of the Computational Singular Perturbation reduction method for chemical kinetics. J. Nonlin. Sci., 14 (2004), 59-91.

[37] A. Zagaris, H.G. Kaper, T.J. Kaper. Fast and Slow Dynamics for the Computational Singular Perturbation Method. Multiscale Model. Sim., 2 (2004), 613-638.

[38] A. Zagaris, H.G. Kaper, T.J. Kaper. Two perspectives on reduction of ordinary differential equations. Math. Nachr., 278 (2005), 1629-1642. 